\title{
Unidades didácticas en física como potenciadores del aprendizaje en estudiantes de ingeniería
}

\author{
Francy N. Jimenez ${ }^{1,2^{\star}}$, Ligia Beleño ${ }^{3}$, Jairo de J. Agudelo ${ }^{1,2}$ y Jorge L. Muñiz \\ (1) Departamento de Física y Matemática, Universidad Autónoma de Manizales, Manizales, Colombia \\ (correo-e: francy@autonoma.edu.co). \\ (2) Departamento de Física y Química, Universidad Nacional de Colombia - Sede Manizales \\ (correo-e: jdjac945@autonoma.edu.co). \\ (3) Departamento de Matemáticas y Ciencias Naturales, Universidad Autónoma de Bucaramanga, Colombia \\ (correo-e: Ibeleno@unab.edu.co). \\ (4) Facultad de Ciencias Básicas, Universidad Tecnológica de Bolívar, Cartagena, Colombia \\ (correo-e: jmuniz@utb.edu.co).
}

${ }^{*}$ Autor a quien debe ser dirigida la correspondencia.

Recibido Mar. 17, 2020; Aceptado May. 18, 2020; Versión final Jul. 27, 2020, Publicado Dic. 2020

\begin{abstract}
Resumen
El objetivo del presente trabajo fue diseñar e implementar unidades didácticas en temas de física clásica y medir su impacto en los procesos de aprendizaje de estudiantes de ingeniería. El diseño de las unidades didácticas partió del conocimiento de ideas previas y de la identificación de los estilos de aprendizaje de los estudiantes. Se definieron los objetivos, los contenidos y las actividades de aprendizaje y de evaluación para potenciar las preferencias por los distintos estilos de aprendizaje. La implementación se llevó a cabo con estudiantes de ingeniería de cuatro ciudades colombianas. Se encontró que el uso de las unidades didácticas favoreció el desarrollo de los estilos de aprendizaje reflexivo y pragmático, y no solo el teórico, identificado como preferente. Se concluye que el uso de esta metodología favoreció la participación de los estudiantes, el conocimiento y potenciación de sus estilos de aprendizaje, el trabajo en equipo, el desarrollo de habilidades comunicativas y el trabajo autónomo y responsable.
\end{abstract}

Palabras clave: unidad didáctica; estilos; aprendizaje; ideas previas; física

\section{Didactic units in physics as learning enhancers for engineering students}

\begin{abstract}
The aim of this study was to design and implement teaching units in classical physics and to measure their impact on the learning processes of engineering students. The design of the didactic units was based on knowledge of previous ideas and on the identification of student learning styles. The objectives, the contents, and the learning and evaluation activities were defined to enhance preferences for different learning styles. This was implemented with engineering students from four Colombian cities. The results show that using didactic units favoured the development of reflexive and pragmatic learning styles, and not exclusively theoretical learning styles, which were identified as preferred. The use of this methodology favoured student participation, knowledge and empowerment of student learning styles, teamwork, communication skills development, and autonomous and responsible work.
\end{abstract}

Keywords: didactic unit; learning styles; previous ideas; physics 


\section{INTRODUCCIÓN}

De acuerdo con investigaciones reportadas (Bigozzi et al., 2018, Collazos y Castrillón, 2016) y la experiencia docente e investigativa de los autores está claramente identificada la dificultad que presentan los estudiantes para alcanzar aprendizajes significativos sobre conceptos físicos, especialmente por su estrecha relación con la matemática. Surge entonces la pregunta de investigación: ¿Cómo diseñar unidades didácticas que favorezca el aprendizaje de conceptos físicos en estudiantes de ingeniería si se busca la articulación entre los conceptos físicos, la modelación matemática y las aplicaciones?

Son diversos los trabajos de investigación enfocados en el estudio de modelos didácticos para la enseñanza de las ciencias naturales (García et al., 2016, Shaw, 2020). Estos modelos didácticos se constituyen en la conexión entre la tradición educativa, relacionada con las producciones teóricas de carácter pedagógico, psicológico, sociológico y curricular; los materiales didácticos; las experiencias prácticas de grupos innovadores; y las actuaciones concretas de profesores en sus aulas (Beier et al., 2019; Alyahyan y Dustegor, 2020). Uno de estos recursos, que ha tomado fuerza en los últimos veinte años, es la unidad didáctica (UD) (Marchán y Sanmarti, 2015) dado que se constituye en un pretexto para que los profesores investiguen y hagan reflexiones sobre su propia práctica y mejoren su desempeño profesional.

El diseño de UD requiere de una planeación relacionada con varios elementos: los objetivos de la enseñanza, los contenidos a desarrollar, las actividades de aprendizaje, las estrategias de enseñanza y las actividades de evaluación (SanMarti, 2002). Además de estos elementos, es importante considerar la forma en que los estudiantes aprenden, la conciencia que tiene sobre las características particulares de sus estilos de aprendizaje preferentes (Al Shaikh et al., 2019) y la consideración de los niveles de preferencia por los estilos, por parte de los docentes, en el diseño de las actividades de aprendizaje, de tal forma que se potencien las capacidades de los estudiantes (Aldusin, 2016). Otro aspecto relevante en el desarrollo de UD es la identificación de las ideas previas. Los estudiantes al enfrentarse a la instrucción formal tienen sus propias concepciones sobre los fenómenos naturales que se van a enseñar en el aula, es decir, tienen esquemas mentales previos (Prosser et al., 2000). Estos esquemas permiten al sujeto interpretar la información que se está enseñando e interfieren decisivamente en la adquisición de conceptos científicos. El conocimiento de las ideas previas de los estudiantes llevará al docente a planear las UD de tal forma que apunten a lograr el, tan anhelado, cambio conceptual (Nadelson et al., 2018).

De otra parte, incluir herramientas tecnológicas en las clases con la intencionalidad adecuada hará unas UD más flexibles (Bloom y Hough, 2003; Ausin et al., 2016; Fallon, 2019) y ayudará a elevar los niveles de preferencia por los estilos de aprendizaje de los estudiantes, ya que proporcionan una mayor posibilidad de emplear los diferentes registros de representaciones (Toro et al., 2012). En concordancia con lo anterior, Bloom y Hough (2003) establecen que se requiere un cambio en la manera de enseñar de tal forma que se aprovechen las habilidades tecnológicas de los estudiantes. Lo anterior, requiere un diseño intencionado del material, así como su revisión permanente, para lo cual es importante conocer las necesidades académicas; buscar enfoques dinámicos, agradables y sencillos; y propender por la participación de los estudiantes. De esta manera, se espera que el material diseñado contribuya en el proceso de aprendizaje del estudiante y que este se vea motivado a emplearlo e interactuar con él para lograr un aprendizaje significativo.

Este trabajo de investigación inició con la identificación y el análisis de las ideas previas de los estudiantes frente a una temática en particular, a partir de la aplicación de un test de entrada. Adicionalmente, se identificaron las preferencias de los estudiantes en los diferentes estilos de aprendizaje a partir de los resultados del test de estilos de aprendizaje CHAEA (Cuestionario Honey-Alonso de Estilos de Aprendizaje) (Alonso et al., 2012). A partir de los análisis de los datos arrojados en la aplicación de los test de entrada y del test CHAEA se desarrollaron UD para los temas: cinemática, dinámica, movimiento oscilatorio, y movimiento ondulatorio. Las UD incluyeron las siguientes actividades: motivación, discusión, ejercicios resueltos, ejercicios propuestos, lecturas complementarias, objetos de aprendizaje, actividades con simuladores, laboratorios virtuales, laboratorios presenciales y desarrollo de proyectos. El objetivo de estas actividades fue promover el cambio conceptual y fortalecer el aprendizaje de los temas: propuesta metodológica que ha sido ampliamente usada en otros contextos (Mirna y Gutiérrez, 2010). Finalmente, se realizó el test después de la instrucción (test de salida) para explorar el cambio de las ideas previas y se aplicó un test con escala Likert con el fin de conocer las apreciaciones de los estudiantes acerca de las UD desarrolladas.

Las UD fueron empleadas durante varios semestres con estudiantes de instituciones educativas que hacen parte de la Red Universitaria Mutis. Como resultado del uso de estas unidades, se encontró que mejoró la disposición de los estudiantes frente a las clases, quienes consideraron que el uso de las UD favoreció sus procesos de aprendizaje y las actividades diseñadas les ayudaron a superar algunas de sus dificultades detectadas. 


\section{OTROS ANTECEDENTES}

A continuación, se presentan algunos referentes teóricos que se tuvieron en cuenta en el desarrollo de este trabajo. Se inicia con el concepto de UD, seguidamente, se describe el marco en el cual se entienden las ideas previas, tan importantes para el desarrollo de esta investigación, y finalmente se hace mención a los estilos de aprendizaje según la teoría de Honney Alonso.

\section{Unidades didácticas}

Una UD se entiende como "un proceso flexible de planificación de la enseñanza de los contenidos relacionados con un campo del saber específico para construir procesos de aprendizaje en una comunidad determinada" (SanMarti, 2002). En otras palabras, es una secuencia de enseñanza "completa" en términos del desarrollo curricular de un objeto determinado, como puede ser un tema o un proyecto. Estas actividades de enseñanza deben estar organizadas y seguir alguna estratégica didáctica, es decir, no son actividades dispuestas al azar, sino que están orientadas de manera intencional. Las estrategias de enseñanza de una UD se manifiestan a través de la lectura didáctica de las secuencias o ciclos de enseñanza que incluyen; debe ser posible identificar la naturaleza de las actividades propuestas e interpretar la intencionalidad de cada una de ellas en relación con el contexto de que forma parte (SanMarti, 2002).

En el diseño de las UD deben tomarse decisiones relacionadas con los objetivos, los contenidos, la organización y secuenciación de los contenidos, las actividades de enseñanza y de evaluación, y la organización y gestión del aula (Romero y Segovia, 2001). Los objetivos, por ejemplo, se van precisando a medida que se definan los contenidos. Para la secuenciación de contenidos pueden emplearse herramientas como esquemas, tramas de contenido que permitan encontrar la interrelación de estos, y mapas conceptuales (Cañas, 2016; Coma et al., 2018). Las actividades incluyen la forma como se enseña y se aprende: no es la actividad concreta la que posibilita aprender, sino el proceso completo diseñado, es decir, el conjunto de actividades organizadas y secuenciadas que posibilitan la interacciones entre los profesores, los estudiantes y el material didáctico.

\section{Ideas previas}

Según la teoría de ideas previas, los estudiantes llegan a las clases de ciencias con una variedad de ideas previas -muchas veces alejadas del conocimiento científico- relacionadas con conceptos necesarios para interpretar los fenómenos naturales que les rodean (Taberk, 2019). Entre sus características se resalta que están presentes de manera semejante en distintas edades, género y culturas; son implícitas, puesto que en muchos casos los estudiantes no son conscientes de ellas; estas ideas son contradictorias, puesto que una misma persona es capaz de explicar desde varios puntos de vista, muchas veces inconsistentes entre sí, un mismo fenómeno; son persistentes, es decir, no se modifican fácilmente por medio de la enseñanza tradicional de la ciencia, incluso cuanto la instrucción es reiterada (Mirna y Gutiérrez, 2010). Las ideas previas son compartidas por grupos heterogéneos de personas y por ello adquieren un carácter transcultural y universal. Este fenómeno se manifiesta en los estudiantes de una misma aula, por tanto, estos patrones pueden ser abordados e intervenidos por los profesores a través de diversas estrategias didácticas con la intención de favorecer el cambio conceptual (Lin et al., 2016; Yang y Kuo, 2020).

\section{Estilos de aprendizaje según el modelo Honey, Alonso y Gallego}

Kolb (2014) plantea que la supervivencia de los seres humanos depende de la habilidad para adaptarse a las condiciones cambiantes del mundo, por tanto, propone que cada individuo enfoca el aprendizaje de una forma particular y define el estilo de aprendizaje como un grupo de capacidades para aprender. Estas capacidades se destacan por encima de otras como resultado de la inteligencia, las experiencias vitales propias y las exigencias del medio. Alonso et al. (2012) se refieren a los estilos de aprendizaje como una descripción de actitudes y comportamientos que determinan la forma preferida de aprender de los individuos; por ello hablaron de preferencias de estilos de aprendizaje por ser una clasificación más precisa que la dada por el concepto de estilo.

El modelo desarrollado por Alonso et al. (2012) define el aprendizaje como un ciclo que se produce en cuatro etapas: experiencia, reflexión sobre la experiencia, formulación de hipótesis y aplicación de las hipótesis. Lo ideal, afirman los autores, es que las cuatro etapas se manifestaran de forma equilibrada en cada sujeto, pero lo cierto es que los individuos interiorizan una determinada etapa del ciclo lo cual define su estilo de aprendizaje predominante: activo, reflexivo, teórico o pragmático. Un instrumento utilizado para el diagnóstico de los estilos de aprendizaje según este modelo es el Learning Style Questionnarie (LSQ). Las respuestas a este cuestionario son un punto de partida y no un final, pues se utilizan para diseñar acciones que potencien las preferencias por aquellos estilos menos sobresalientes en los sujetos, con el fin de aumentar la efectividad del aprendizaje, crear grupos de trabajo productivos y optimizar los talleres de formación para mejorar el desempeño de las personas en las actividades empresariales. 
En la adaptación al ámbito académico y al idioma español del LSQ se agregó un apartado de información socio-académica, se modificó las instrucciones de respuesta y se diseñó la hoja de perfil de aprendizaje (Alonso, et al., 2012). Este test, que se conoce como el Cuestionario Honney-Alonso de Estilos de Aprendizaje (CHAEA), consta de 80 ítems con dos opciones de respuesta cerrada (+y -). El test distribuye a los estudiantes según su grado de preferencia por los cuatro estilos de aprendizaje, cada uno de los cuales está representado en el cuestionario por 20 ítems. Los resultados indican cual es el estilo más usado por el estudiante, no en forma exclusiva sino preferente ya que, en general, todos los estilos están presentes en cada persona.

\section{METODOLOGÍA}

En esta sección se presentan los materiales y los métodos empleados en el desarrollo de la presente investigación la cual se desarrolló básicamente en dos etapas: primero se presenta el diseño de la estrategia y segundo su implementación.

\section{Diseño de instrumentos y unidades didácticas}

El proyecto inició desde el I semestre del año 2013 y se extendió hasta el II semestre de 2016. Se realizó un estudio exploratorio descriptivo para la identificación de las ideas previas con grupos de estudiantes para cada tema en particular así: cinemática y dinámica una muestra de 62 estudiantes de diversas ingenierías en la Universidad Autónoma de Manizales (Jiménez et al., 2015) quienes iniciaban el curso de Física Mecánica; oscilaciones y ondas una muestra de 173 estudiantes de ingeniería pertenecientes a 4 instituciones de educación superior (IES) vinculadas a la Red Universitaria Mutis quienes tomaban en ese momento los cursos de física oscilaciones y ondas, y ecuaciones diferenciales. Para este estudio de ideas previas, se diseñaron y aplicaron varios test que permitieron identificar los obstáculos de aprendizaje frente a estos temas y diseñar actividades que tuvieran en cuenta dichos obstáculos y permitieran potenciar la preferencia por los diferentes estilos de aprendizaje (los test de entrada salida para cada tema se pueden encontrar en https://bit.ly/2ZKPpOb). Para identificar los estilos de aprendizaje se utilizó el test CHAEA el cual permitió conocer las preferencias en sus formas de aprender.

Posteriormente, se diseñaron UD sobre cada temática en las cuales se incorporaron los siguientes elementos: los objetivos que se esperaban alcanzar en el curso; los contenidos desarrollados en diferentes formas, entre ellas empleando objetos de aprendizaje (OA) diseñados previamente (Jiménez et al., 2016); las actividades de aprendizaje planteadas de acuerdo a los diferentes estilos y tomando como punto de partida las ideas previas identificadas, y finalmente las actividades de evaluación; todo ello enmarcado dentro de un proceso de gestión del aula claramente planificado. Esa metodología ha sido empleada anteriormente en investigaciones relacionadas con didáctica de las ciencias (Mirna y Gutiérrez, 2010), pero su uso se ha limitado, aunque no exclusivamente, a nivel de básica primaria, secundaria y media y muy poco a nivel universitario.

\section{Implementación de la metodología}

En el II semestre de 2014 se implementaron las UD de los temas cinemática y dinámica con 62 estudiantes (30 la IES1y 32 de la IES2). En el II semestre del 2016 se implementaron las UD de oscilaciones y ondas con 60 estudiantes (30 de la IES1 y 30 de IES2). Los estudiantes tomaban los cursos de Física I y Física II en sus instituciones respectivas y hacían parte de uno de los cinco cursos que en promedio tiene cada institución por semestre. Es decir, se tomó una muestra representativa de la población de estudiantes en estos cursos para cada semestre. Todos los estudiantes estuvieron inscritos, en la plataforma virtual conjunta y compartieron el material diseñado, sin embargo estuvieron matriculados en grupos independientes. Los docentes que dirigieron los cursos estuvieron en permanente contacto en busca de desarrollar los cursos de forma similar, con las diferencias obvias de tener profesores y entornos distintos.

En las actividades de aprendizaje realizadas se buscaba incluir algunas que favorecieran los niveles de preferencia de los estudiantes por los cuatro estilos de aprendizaje, como se muestra en la Tabla 1. Se tuvieron en cuenta actividades tanto para el aprendizaje como del aprendizaje. Para el aprendizaje, durante el desarrollo de las actividades se realizó una evaluación permanente de la evolución de los estudiantes con la respectiva retroalimentación al trabajo desarrollado. Del aprendizaje, las evaluaciones escritas y los informes finales de los proyectos permitieron determinar el alcance de los objetivos de aprendizaje propuestos.

Cada una de las actividades de aprendizaje diseñadas era retroalimentada mediante alguna forma de evaluación; por ejemplo, se socializaron los videos en foros tanto virtuales como presenciales; se evaluaron las lecturas mediante mapas conceptuales que los estudiantes debían realizar y compartir con sus compañeros; se realizaron las evaluaciones escritas (además del test de entrada salida) y las exposiciones 
para monitorear la solución de los problemas propuestos y las actividades dejadas en los OA; se evaluaron los laboratorios presenciales y virtuales, así como los simuladores, mediante informes escritos; finalmente, se realizaron presentaciones orales y escritas de los proyectos integradores desarrollados en grupos de trabajo. Mediante estas formas de evaluación, fue posible monitorear el proceso de evolución conceptual en los estudiantes.

Tabla 1. Actividades desarrolladas para los 4 estilos de aprendizaje

\begin{tabular}{|c|c|c|c|}
\hline Estilo Reflexivo & Estilo Teórico & Estilo Activo & Estilo Pragmático \\
\hline $\begin{array}{l}\text { Videos con preguntas } \\
\text { motivacionales }\end{array}$ & $\begin{array}{l}\text { Talleres de preguntas } \\
\text { teóricas }\end{array}$ & $\begin{array}{l}\text { Laboratorios virtuales y } \\
\text { presenciales }\end{array}$ & \multirow{2}{*}{$\begin{array}{l}\text { Desarrollo de un Proyecto } \\
\text { integrador }\end{array}$} \\
\hline $\begin{array}{l}\text { Foros y lecturas para } \\
\text { reflexionar }\end{array}$ & Solución de problemas & Simuladores & \\
\hline
\end{tabular}

\section{RESULTADOS Y DISCUSIÓN}

Teniendo en cuenta el objetivo de determinar el impacto del uso de las UD previamente diseñadas con estudiantes de ingeniería, los resultados se agruparon en torno a tres aspectos: los entregables de cada tipo de actividad de aprendizaje, los resultados de las pruebas vs estilos de aprendizaje, y la apreciación de los estudiantes frente a la metodología empleada. Después de la presentación de los resultados se incluye una sección de discusión de los resultados en forma separada para enfatizar los logros alcanzados.

\section{Los entregables en cada tipo de actividad de aprendizaje}

La implementación de las UD inició dando a conocer a los estudiantes los objetivos de aprendizaje al comienzo de cada tema particular. De esta manera fue posible realizar un seguimiento a los avances de los estudiantes en función de su cumplimiento. Los OA se constituyeron en herramientas valiosas para que los estudiantes repasaran los contenidos permanentemente. Algunos de estos objetos pueden encontrarse en (http://fismat.autonoma.edu.co/OASonido/,http://fismat.autonoma.edu.co/MovimientoOscilatorio/,http://fismat. autonoma.edu.co/MovimientoOndulatorio/). Las actividades de aprendizaje y de evaluación se desarrollaron tanto en forma virtual (desde la plataforma de los cursos y los OA) como presencial (exposiciones, sustentaciones y evaluaciones escritas). De acuerdo con las actividades planteadas para los distintos estilos de aprendizaje, se realizaron análisis cualitativos y cuantitativos de los entregables de los estudiantes. Estos entregables se agrupan por actividades propuestas de acuerdo con cada estilo de aprendizaje, como se describe a continuación.

\section{Fortalecer los niveles de preferencia por el estilo reflexivo}

Participación en foros: se llevarón a cabo dos foros, uno para cada unidad didáctica, en los cuales se plantearon interrogantes a los que los participantes debían responder. Su objetivo era que los estudiantes se documentaran alrededor del tema y que plantearan su opinión de manera escrita mediante un documento breve con sentido y pertinencia. Los docentes se hicieron cargo del manejo de los foros direccionando las discusiones, motivándo a los estudiantes a participar y a tener en cuenta los aportes de los demás compañeros.

Mapas conceptuales de las lecturas complementarias: en las UD se sugirieron lecturas complementarias de los temas que incluían aplicaciones en diversas áreas relacionadas con cuestiones de su cotidianidad. Su objetivo era que el estudiante identificara la idea principal, sus características y aplicaciones, y compartieran con sus compañeros una reflexión de la lectura. Para ello, debían hacer uso de mapas conceptuales que ellos mismos construyeron. Al finalizar las actividades, los estudiantes expresaron que el mapa conceptual les facilitó la comprensión y la socialización de los temas, resultado que ha sido encontrado en otras investigaciones (Coma, 2018). En el siguiente enlace se encuentran algunos ejemplos de mapas conceptuales construidos por los estudiantes (https://bit.ly/3dZHL7L).

\section{Fortalecer los niveles de preferencia por el estilo activo}

Se adaptaron laboratorios virtuales existentes en la red para los cuales se estructuraron guías de trabajo que permitieran la comparación de resultados respecto a los laboratorios presenciales. Además, se diseñaron e implementaron simuladores en Geogebra para fortalecer el manejo gráfico y vectorial de las cantidades físicas; en las Figuras 1 y 2 se muestran algunos ejemplos de estos simuladores. Las guías contenían una descripción de las actividades que debían desarrollar, tanto con los simuladores como con los laboratorios virtuales, de las cuales debían entregar un informe escrito. El uso de simuladores como los diseñados en este 
trabajo ha sido empleado en otras investigaciones, por ejemplo, Collazos y Castrillón (2016) realizaron simulación sobre el movimiento oscilatorio y las emplearon con estudiantes universitarios, encontraron que el uso de estas herramientas ayudó a mejorar el nivel de satisfacción de los estudiantes frente a este tema.

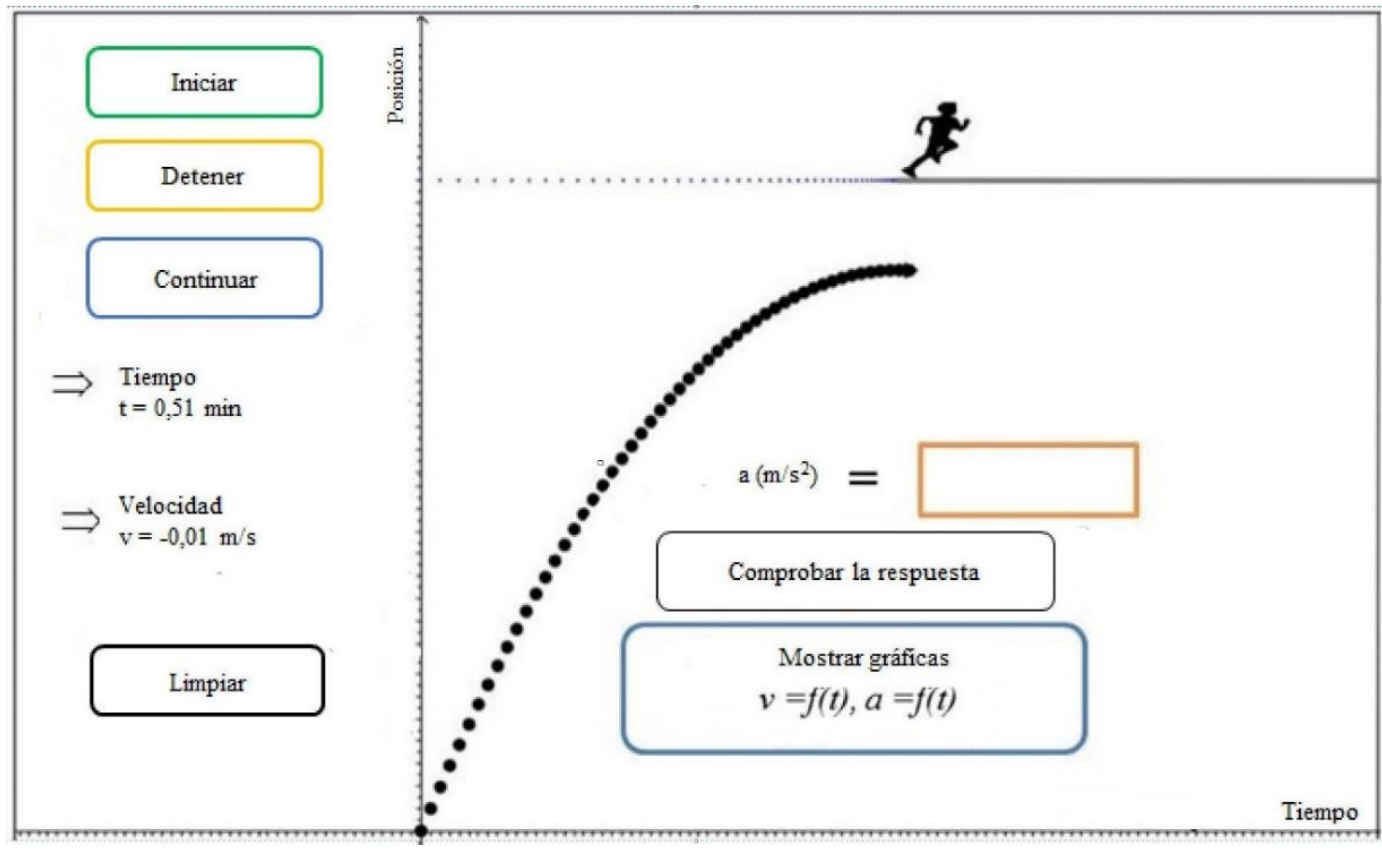

Fig. 1: Ejemplos simulador cantidades cinemáticas

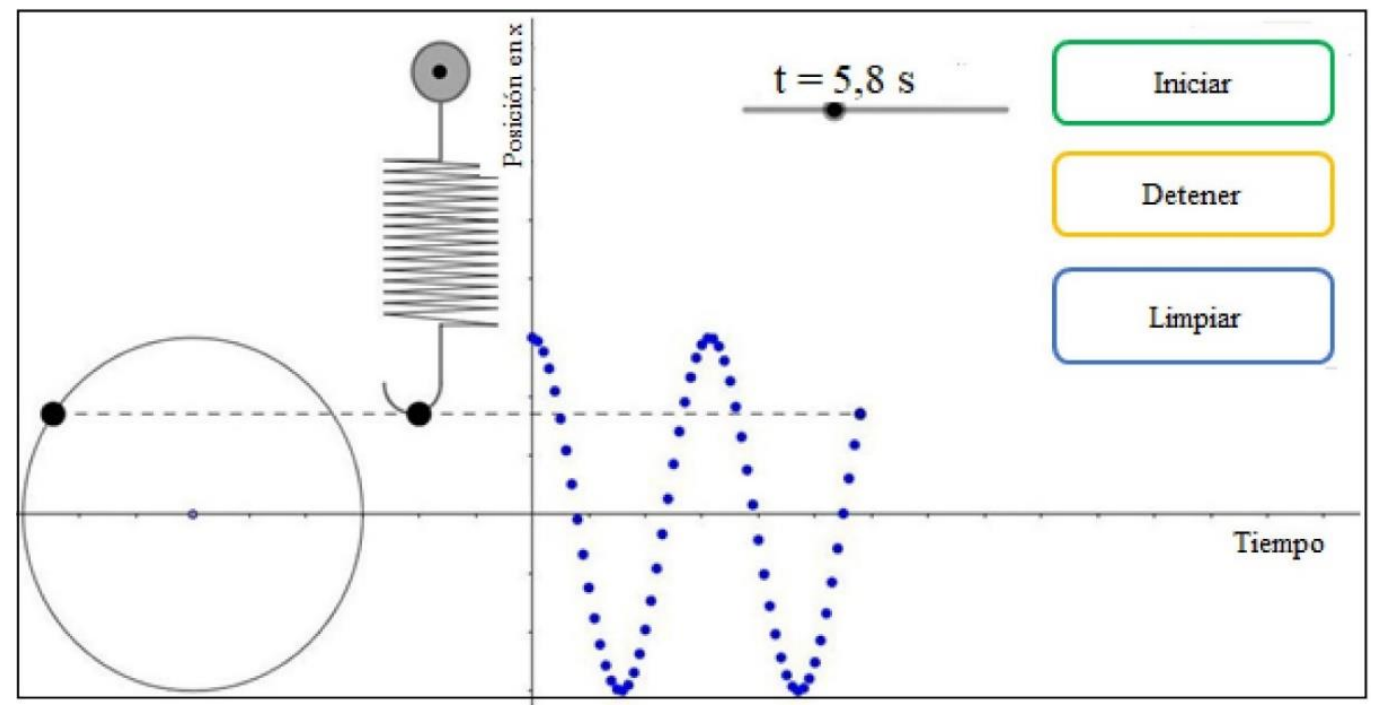

Fig. 2: Ejemplos simulador movimiento oscilatorio

\section{Fortalecer los niveles de preferencia por el estilo teórico}

Estas actividades requerían que el estudiante comprendiera un problema planteado, lo asociara con ejemplos y temas de clase, y planteara una estrategia de solución. Además, se buscaba favorecer las habilidades comunicativas: debían exponer en clase el problema abordado, empleando un tiempo limitado y haciendo uso de diagramas, dibujos, ecuaciones y el material que consideraran conveniente para socializar la solución encontrada. Se realizaron, además, discusiones en torno a preguntas teóricas propuestas en los talleres en las cuales se observó una participación masiva de los estudiantes.

\section{Fortalecer los niveles de preferencia por el estilo pragmático}

Su objetivo fue el diseño e implementación de un proyecto relacionado con uno de los temas desarrollados en clase; para el caso de Física I, se propuso el análisis de un sistema cotidiano, como el funcionamiento de una bicicleta, desde los conceptos cinemático tanto traslacionales como rotaciones, así como el análisis dinámico. Para el caso de física II, se trabajó una aplicación particular, el Yugo Escocés, en la cual se buscaba 
que estudiaran las características cinemáticas para posteriormente diseñar un prototipo. De acuerdo con la guía del proyecto, explicada el primer día de clase, los estudiantes plantearon una propuesta de aplicación de uno de estos mecanismos y lo diseñaron e implementaron a escala piloto. Este trabajo fue realizado en grupos de 3 estudiantes y debían presentar avances en tres momentos: primero al profesor, quien realizaba la retroalimentación respectiva; después a sus compañeros en una exposición concisa; y, finalmente, en un escrito en formato tipo artículo. El uso de proyecto como metodología de enseñanza fue empleado en este trabajo ya que reportes de la literatura muestran que es una estrategia con mayor eficacia frente a los logros académicos de los estudiantes que los métodos tradicionales de enseñanza (Ayaz y Söylemez., 2015). En las Figuras 3 y 4 se presentan algunos de los proyectos desarrollados por los estudiantes al igual que en el enlace https://bit.ly/2W86scn.

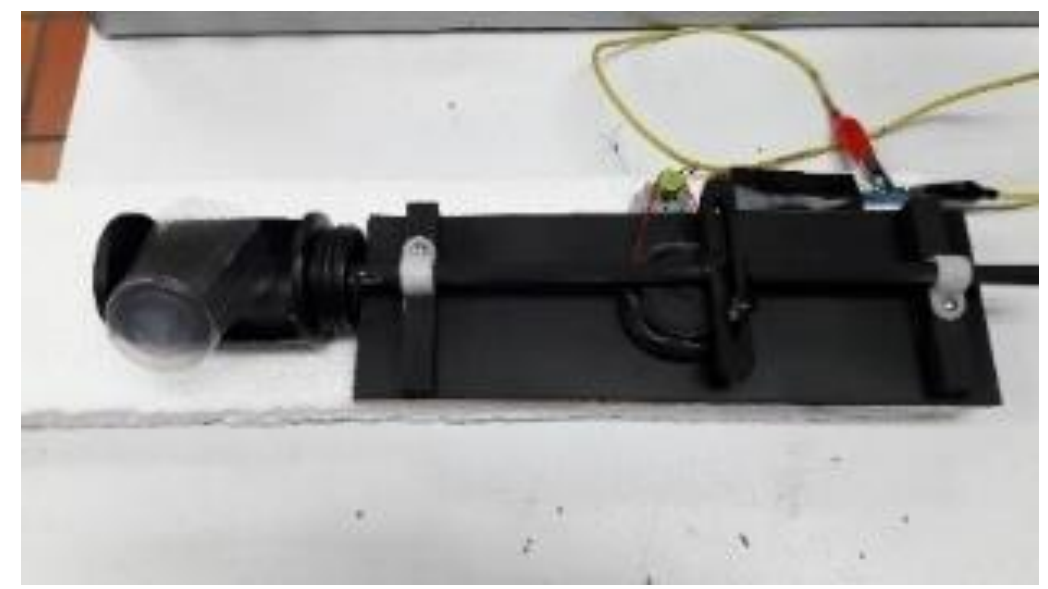

Fig. 3: Muestra del proyecto integrador: Compresor de latas

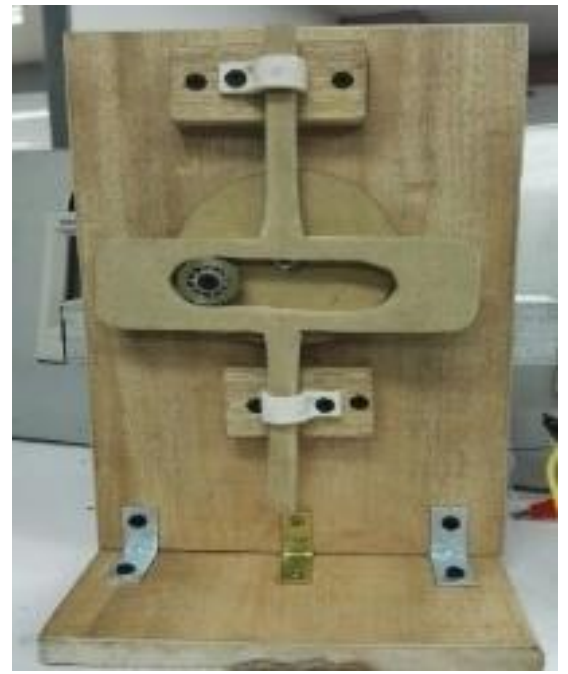

Fig. 4: Muestra del proyecto integrador: Máquina de sello

Se realizó una prueba de hipótesis para la diferencia de proporciones entre las respuestas correctas en el test de entrada y salida para cada grupo. En la Tabla 2 se presentan las proporciones de acierto y los $p$-valores obtenidos. Estos resultados indican que las actividades propuestas permitieron que los estudiantes lograran una evolución conceptual desde sus ideas previas, además que alcanzaran niveles de desempeño mayores a aquellos estudiantes que no tuvieron la oportunidad de trabajar los temas con esta metodología como se analiza de manera detalla en trabajos anteriores (Jiménez et al., 2015; Jiménez et al., 2019).

Tabla 2. Resultados prueba de hipótesis para la diferencia de proporciones del test entrada salida

\begin{tabular}{|l|c|c|}
\hline & Curso de Física I, semestre II de 2013 & Curso de Física II, semestre II de 2016 \\
\hline \% de acierto en el Test de entrada & 0,384 & 0,328 \\
\hline \% de acierto en el Test de salida & 0,502 & 0,497 \\
\hline p-valor diferencia de proporciones & $1 \times 10^{-4}$ & $6 \times 10^{-7}$ \\
\hline
\end{tabular}




\section{Resultados de las pruebas versus estilos de aprendizajes}

Se realizó una identificación de los estilos de aprendizaje a partir test CHAEA a los 122 estudiantes participantes en el estudio. Los resultados se presentan a continuación. Se encontró que el $39,62 \%$ de los estudiantes mostró preferencia por un solo estilo de aprendizaje, siendo el teórico el predominante en el 22,64 $\%$ de la población. El estilo de aprendizaje activo resultó predominante en el $13,21 \%$ y los estilos de aprendizaje reflexivo y pragmático resultaron predominantes en un 1,89\%. Del total de estudiantes, el 18,86 $\%$ mostró preferencias por dos estilos de aprendizaje, las mayores proporciones se dieron en combinación con el estilo el teórico. El 13,2\% mostró preferencias por tres estilos de aprendizaje y el 9,43\% mostró preferencias por los cuatro estilos de aprendizaje.

La planeación y desarrollo de las UD se realizó de acuerdo con el ciclo de aprendizaje experiencial de Honey y Alonso. Con el objetivo de favorecer la diversidad de preferencias a la hora de percibir y procesar la información, se implementaron una serie de actividades que cubrían el ciclo de aprendizaje completo. Para medir la efectividad de estas actividades de aprendizaje, de acuerdo con el estilo de mayor preferencia, se agruparon las notas dadas a las actividades en cuatro tipos de evaluaciones, las cuales se denotaron como prueba 1 para el estilo reflexivo, prueba 2 para el estilo teórico, prueba 3 para el estilo activo y prueba 4 para el estilo pragmático. La calificación obtenida por los estudiantes en cada una de estas pruebas se cruzó contra su preferencia en cada estilo. Las calificaciones obtenidas se agruparon en tres categorías de rendimiento académico: bajo de 0 a 2,90, básico de 3,00 a 3,90 y alto de 4,00 a 5,00, a partir de las cuales se estimó la correlación entre los estilos de aprendizaje y el rendimiento académico en las 4 pruebas a un nivel de confianza del $95 \%$ y significancia de 0,05 .

En la Tabla 3 se presentan los resultados de los porcentajes de correlación y sus respectivos p-valores. Se evidencia que existe una correlación significativa entre el grado de preferencia por el estilo de aprendizaje reflexivo y el resultado de la prueba 1 , y entre el grado de preferencia por el estilo de aprendizaje pragmático y el resultado de la prueba 4. Ambas correlaciones son bajas de acuerdo con los rangos propuestos por Cohen (1998). En su investigación de López y Silva (2009) encontraron que la preferencia por el estilo reflexivo tiene relación con el rendimiento académica, también con correlaciones bajas, lo que es acorde al resultado anterior. De otra parte, los autores también establecieron que la preferencia por los estilos activo y pragmático disminuyen con el paso de los años escolares, lo que permite entender que en este trabajo el estilo pragmático presente correlación con el rendimiento ya que los estudiantes están aún en niveles iniciales de su formación.

Tabla 3. Resultados de las correlaciones entre estilos de aprendizaje y las evaluaciones para cada estilo

\begin{tabular}{|l|c|c|c|c|}
\hline & Reflexivo vs prueba 1 & Activo vs prueba 2 & Teórico vs prueba 3 & Pragmático vs prueba 4 \\
\hline $\begin{array}{l}\text { Coeficiente de } \\
\text { Correlación }\end{array}$ & 0,301 & 0,261 & $-0,017$ & 0,338 \\
\hline Significancia & 0,036 & 0,070 & 0,905 & 0,018 \\
\hline
\end{tabular}

Posteriormente, se promedió la nota de las cuatro pruebas aplicadas a los estudiantes durante el desarrollo de las UD. Se encontró que, a niveles de confianza del $99 \%$ y significancia de 0,01 bilateral, existe una correlación significativa entre el grado de preferencia por el estilo de aprendizaje reflexivo y el rendimiento académico promedio. El coeficiente de correlación en este caso fue de 0,406, por tanto se considera una correlación moderada (Cohen, 1988). Estos resultados están en coherencia con los encontrados por Alducin y Vásquez (2016) quienes indican que el estilo reflexivo presenta correlación con los resultados en matemáticas para los estudiantes universitarios incluidos en su estudio.

\section{Apreciación de los estudiantes frente a la metodología empleada}

Con el fin de conocer el grado de aceptación de las UD por parte de los estudiantes, se diseñó y aplicó un cuestionario con escala Likert. El test consistió en 8 afirmaciones relacionadas con los procesos de enseñanza y aprendizaje empleando las UD, de las cuales los encuestados debían seleccionar una opción, Para tal elección, debían tener en cuenta la escala de valores, en la cual 5 es el puntaje positivo más alto y 1 el negativo más bajo, así: 5 totalmente de acuerdo (TA), 4 de acuerdo (A), 3 indeciso (I), 2 en desacuerdo (D) y 1 totalmente en desacuerdo (TD).

En la Tabla 4 se presenta el consolidado del porcentaje en las respuestas obtenidas. La columna designada como "promedio" se obtuvo dividiendo el puntaje total de cada pregunta sobre el número de estudiantes que la respondieron y la columna denominada como "porcentaje" mediante la división entre el puntaje total de cada pregunta y el puntaje máximo posible que es 320 (64 estudiante x 5 puntaje máximo posible/estudiante). Se presentan los resultados con los datos tomados de 64 de los 122 estudiantes participantes en el estudio. 
Se observa que la pregunta con menor promedio y porcentaje es la P1, la cual indagaba por el uso que habían hecho los estudiantes de UD en otras áreas de las ciencias naturales, lo cual indica que esta no es una metodología de uso frecuente. A continuación, se describen los resultados de cada pregunta en términos de la escala de valores establecida.

En la pregunta P1 el 48,4\% de los estudiantes expresó haber empleado UD similares a los usadas en este curso, frente a un 17,2 \% que manifestó no haberlas usado y un 34,3 \% que eligió la opción "indeciso". Lo anterior indica que solo en algunos cursos se emplean UD con frecuencia y que los estudiantes no están acostumbrados a emplear esta metodología. En la pregunta P2 se indagó por el conocimiento que tiene los estudiantes sobre lo que es una UD. Un 81,5\% (entre A y TA) de los estudiantes manifestó entender lo que es una UD, frente a un 7,8 \% que manifestó no saberlo (TD) y un $11 \%$ que eligió la opción "indeciso" la cual se entiende como que no lo tienen del todo claro. Las preguntas P3 y P4 hacían referencia a la facilidad para seguir las UD. El 81,27\% de los estudiantes (los que respondieron las opciones TA y A) consideró que el diseño de las UD es claro, adecuado y que son fáciles de seguir; frente a un $4,7 \%$ que no lo consideró así. De acuerdo con la P5, el 79,7 \% de los estudiantes (los que contestaron las opciones TA y A) consideró que los contenidos de las UD fueron expuestos en forma clara y le permitieron aprender los temas, frente a un 3 $\%$ (los que contestaron las opciones D y TD) que no lo consideró así y un 17,2 \% que estaba indeciso.

Tabla 4: Consolidado de las respuestas al test de apreciación respecto a las UD

\begin{tabular}{|l|l|l|l|l|l|l|l|l|}
\hline Pregunta & 5 & 4 & 3 & 2 & 1 & Puntaje total & Promedio & Porcentaje \% \\
\hline P1 & 8 & 23 & 22 & 10 & 1 & 219 & 3,42 & 68,4 \\
\hline P2 & 18 & 34 & 7 & 4 & 1 & 256 & 4,00 & 80,0 \\
\hline P3 & 9 & 44 & 9 & 1 & 1 & 251 & 3,92 & 78,4 \\
\hline P4 & 13 & 38 & 9 & 3 & 1 & 251 & 3,92 & 78,4 \\
\hline P5 & 9 & 42 & 11 & 1 & 1 & 249 & 3,89 & 77,8 \\
\hline P6 & 7 & 41 & 9 & 5 & 2 & 238 & 3,72 & 74,4 \\
\hline P7 & 14 & 25 & 15 & 9 & 1 & 234 & 3,66 & 73,1 \\
\hline P8 & 14 & 37 & 10 & 2 & 1 & 253 & 3,95 & 79,1 \\
\hline
\end{tabular}

La pregunta P6 buscaba determinar si los estudiantes consideran que las actividades sugeridas en las UD le ayudaron a desarrollar los diferentes estilos de aprendizaje explorados en el test de Honey Alonso. El $75 \%$ de los estudiantes consideró que las actividades sí favorecieron el desarrollo de los estilos de aprendizaje, frente a un $10,9 \%$ que no lo consideró así y un $14,1 \%$ que se muestra indeciso. La P7 indagaba sobre la percepción del estudiante acerca de la utilidad de esta herramienta para apoyar su aprendizaje. Un 79,7 \% de los estudiantes (los que eligieron las opciones TA y A) consideró que las actividades propuestas en las unidades le permitieron fortalecer el aprendizaje, frente a un 4,7 \% que consideró que no fue así y un 15,6\% que estaba indeciso. La pregunta P8 preguntaba si le gustaría que todos los temas de curso estuvieran estructurados en UD. Un 60,9\% (los que eligieron las opciones TA y A) manifestó que le gustaría, frente a un $15,6 \%$ que no le gustaría y $23,4 \%$ a que estaba indeciso. Esto puede deberse a que como es una metodología a la que no están muy habituados se genera cierta resistencia a su empleo.

\section{Discusión de los resultados}

De acuerdo con los resultados obtenidos en este estudio sobre el uso de UD para la enseñanza y el aprendizaje de conceptos de física básica con estudiantes de ingeniería se destacan los siguientes aspectos. Primero, en cuanto al diseño e implementación de las UD se deben tener en cuenta elementos como la disposición de los estudiantes, la planificación y gestión de los recursos de la UD por parte del profesor, el seguimiento en la participación de los estudiantes tanto en el aula de clase como a través de los foros, la retroalimentación permanente al trabajo desarrollado y la transposición de lo aprendido mediante aplicaciones particulares de la vida cotidiana y de la ingeniería.

Segundo, en relación con los estilos de aprendizaje se encontró que el estilo teórico resulto ser preferente por esta muestra de estudiantes de ingeniería, dejando de manifiesto la necesidad de potenciar el desarrollo de los demás estilos de aprendizaje. Lo anterior, teniendo en cuenta que se están formando ingenieros que deben desarrollar distintos tipos de habilidades relacionadas no solo con la conceptualización teórica, sino con la experimentación, la aplicación práctica de los conocimientos y la reflexión permanente. Es claro que en las metodologías tradicionales se hace poco énfasis en distintos tipos de evaluación las cuales resultan ser necesarias para los estudiantes ya que favorecen el desarrollo de otros estilos de aprendizaje. El uso de las diversas actividades de aprendizaje y de evaluación mostró que se potencia el desarrollo de los diferentes estilos de aprendizaje; en este trabajo, se observan mejoras significativas en los desempeños de los estudiantes con estilos reflexivo y pragmático que en una metodología tradicional no sería posible. Lo anterior 
indica que el conocimiento de los estilos de aprendizaje de los estudiantes, por parte tanto de los docentes como de los mismos estudiantes, es un elemento importante cuando se busca mejorar los procesos de enseñanza y aprendizaje.

Tercero, en cuanto a la percepción de los estudiantes se puede afirmar que el grado de aceptación de los estudiantes frente a esta metodología fue adecuado, aunque es importante anotar que algunos de ellos muestran cierta resistencia a su implementación, ya que es una metodología a la cual no están habituados y que requiere compromiso y esfuerzo adicional para participar y desarrollar las actividades propuestas. Además, son necesarios unos niveles de reflexión, responsabilidad y autonomía en su proceso de aprendizaje que algunos estudiantes aún no alcanzan, aunque otros si logran desarrollar estrategias autorreguladoras de sus propias capacidades, que les permiten superar obstáculos en el aprendizaje de los conceptos físicos y los modelos matemáticos asociados. Se notó que cuando los estudiantes emplean las UD en un segundo curso son más receptivos y dispuestos puesto que se rompe la inercia que traen de la enseñanza tradicional.

\section{CONCLUSIONES}

De acuerdo con los resultados de este estudió, la discusión presentada y la contrastación con otros autores expuesta anteriormente se presentan las siguientes cuatro conclusiones: 1) Para un adecuado diseño de las UD es importante tener en cuenta el estado inicial de los estudiantes, es decir, la identificación de los obstáculos de aprendizaje para afrontar los temas. En este caso, se partió de la identificación de las ideas previas de los estudiantes y a partir de la identificación de los obstáculos de aprendizaje se estructuró el proceso de enseñanza desde el planteamiento de los objetivos y el desarrollo sistemático de los contenidos y fue guiado por una serie de actividades de aprendizaje intencionalmente desarrolladas para promover la evolución conceptual; 2) Los objetos de aprendizaje, los simuladores y laboratorios virtuales se convierte en materiales interactivos al alcance de los estudiantes en cualquier momento y lugar, los cuales ayudan al desarrollado de habilidades de modelación y experimentación y favorecen el proceso de enseñanza desde la perspectiva del profesor y el proceso de aprendizaje desde la mirada de los estudiantes; 3) El desarrollo de los proyectos integradores resulta ser una actividad valiosa dado que permite una activa y comprometida participación de los estudiantes, favorece el trabajo colaborativo, promueve el pensamiento científico y logra la integración de los conceptos, la formulación matemática y las aplicaciones que resultan ser tan importantes en ingeniería; y 4) Con esta metodología se logró despertar interés en los estudiantes por el aprendizaje de temas como cinemática, dinámica, movimiento oscilatorio y ondulatorio; se les permitió conocer y potenciar sus estilos de aprendizaje; se favorecieron habilidades para el trabajo en equipo, la comunicación de resultados y el trabajo autónomo y responsable.

\section{AGRADECIMIENTOS}

Los autores agradecen a las instituciones de la Red Universitaria Mutis que apoyaron el desarrollo de este proyecto tanto en los recursos para los investigadores como en la participación de los estudiantes. También agradecen a Colciencias por el financiamiento de un joven investigador que apoyo una primera etapa de esta investigación.

\section{REFERENCIAS}

Al Shaikh A., Aldarmahi A. A. y otros cinco autores, Learning Styles and Satisfaction with Educational Activities of Saudi Health Science University Students, doi.org/10.1016/j.jtumed.2019.07.002, Journal of Taibah University Medical Sciences, 14 (5), $418-424$ (2019)

Alducin-Ochoa J.M. y Vázquez-Martínez A.I., Autoevaluación de Conocimientos Previos y Rendimiento según Estilos de

Aprendizaje en un Grado Universitario de Edificación, dx.doi.org/10.4067/S0718-50062016000200004, Formación Universitaria, 9(2), 29-40, (2016)

Alyahyan, E. y Düştegör, D., Predicting Academic Success in Higher Education: Literature Review and Best Practices, doi.org/10.1186/s41239-020-0177-7, International Journal of Educational Technology in Higher Education, 17(3), 1-21 (2020)

Alonso, C., Gallego D. y Honey P., Los estilos de aprendizaje: Procedimientos de diagnóstico y mejora, ISBN: 978-84271-1914-7, Ediciones Mensajero, 7a edición, Bilbao, España (2006)

Ausín, V., Abella, V. y otros dos autores, Aprendizaje Basado en Proyectos a través de las TIC: Una Experiencia de Innovación Docente desde las Aulas Universitarias, dx.doi.org/10.4067/S0718-50062016000300005, Formación Universitaria, 9(3), 31-38 (2016)

Ayaz, M.F. y Söylemez, M.E., The Effect of the Project-based Learning Approach on the Academic Achievements of the Students in Science Classes in Turkey: A Meta-analysis Study, doi.org/10.15390/EB.2015.4000, Egitim ve Bilim, 40(178), 255-283 (2015) 
Beier, M.E., Kim, M.H. y otros cuatro autores, The Effect of Authentic Project-based Learning on Attitudes and Career Aspirations in STEM, doi.org/10.1002/tea.21465, Journal of Research in Science Teaching, 56(1), 3-23 (2019)

Bigozzi, L., Tarchi, C. y otros dos autores, The Influence of Teaching Approach on Students' Conceptual Learning in Physics, doi.org/10.3389/fpsyg.2018.02474, Frontiers in Psychology, 9(2474), 1-14 (2018)

Bloom, K.C. y Hough, M.C., Student Satisfaction with Technology-Enhanced Learning, CIN: Computers, ISSN: 1538-2931, Informatics Nursing, 21(5), 241-246 (2003)

Cañas F, Lazo L. y Carcamo C., Chemistry Learning with Application of the Zone of Proximal Development and Use of Conceptual Maps in the Chemistry Lab, dx.doi.org/10.4067/S0717-97072016000100002, Journal of the Chilean Chemical Society, 61 (1), 2757-2762 (2016)

Cohen, J., Statistical Power Analysis for the Behavioral Sciences, ISBN 0-8058-0283-5, 2ª edition. Hillsdale, NJ: Erlbaum, (1988)

Collazos, H.F. y Castrillón, O.D., Metodología para la Enseñanza del Movimiento Oscilatorio mediante Simulación Computarizada, dx.doi.org/10.4067/S0718-07642019000400165, Información Tecnológica, 30(4), 165-180 (2019)

Coma-Rosello T., Aguelo-Arguis A. y otros tres autores, Analysis of Innovative Approaches in the Class Using Conceptual Maps and Considering the Learning Styles of Students, doi: 10.1109/RITA.2018.2879388, Revista Iberoamericana de Tecnologías del Aprendizaje, 13 (4), 8532120, 120-129 (2018)

Falloon, G., Using Simulations to Teach Young Students Science Concepts: An Experiential Learning Theoretical Analysis, doi.org/10.1016/j.compedu.2019.03.001, Computers and Education, 135,138-159 (2019)

García, J.D, Urionabarrenetxea, S. y Bañales, A., Cambios en Metodologías Docentes y de Evaluación: ¿Mejoran el Rendimiento del Alumnado Universitario?, Revista Electrónica de Investigación Educativa, ISBN: 16074041 18(3), 1-18 (2016)

Jiménez-García, F.N., Agudelo-Calle, J. J. y Vargas-Sánchez, J.J., Incidencia de la Intervención Didáctica en el Aprendizaje de Conceptos Cinemáticos en Estudiantes de Ingeniería de la UAM Analizada Desde sus Ideas Previas, Revista Educación en Ingeniería, ISSN 1900-8260, 10(19), 26-38 (2015)

Jiménez-García, F. N., Márquez-Narváez, C. y otros cuatro autores, Una Experiencia Didáctica en el Diseño e Implementación de Objetos de Aprendizaje para la Enseñanza de la Física, Revista Educación en Ingeniería, ISSN $1900-$ 8260, 11(22), 13-20 (2016)

Jiménez-García, F. N., Beleño-Montagut L. y otros tres autores, La Evolución Conceptual en el Aprendizaje de Conceptos Físicos Mediada por Unidades Didácticas, Revista Educación en Ingeniería, ISSN 1900-8260, 14(27), 1-8 (2019)

Kolb, D. A., Experiential Learning: Experiences as the Source of Learning Development, ISBN 9780133892505, 2ª edición, Pearson Education, Nueva York, USA (2014)

Link, J. W., Yen M. H. y otros tres autores, Examining the Factors That Influence Students' Science Learning Processes and Their Learning Outcomes: 30 Years of Conceptual Change Research, Eurasia Journal of Mathematics, Science and Technology Education, ISSN: EISSN-1305-8223, 12(9), 2617-2646 (2016)

López-Aguado M. y Silva-Falchetti E., Estilos de Aprendizaje. Relación con Motivación y Estrategias, Revista Estilos de Aprendizaje, ISSN-e 1988-8996, 2(4), 36-55 (2009)

Marchán-Carvajal, I. y Sanmartí, N., Criterios para el Diseño de Unidades Didácticas Contextualizadas: Aplicación al Aprendizaje de un Modelo Teórico para la Estructura Atómica. doi.org/10.1016/j.eq.2015.06.001 Educación Química, 26(4), $267-274(2015)$

Mirna, C. M. y Gutiérrez, O. A., Estrategia de Enseñanza Basada en el Cambio Conceptual para la Transformación de Ideas Previas en el Aprendizaje de las Ciencias, doi:10.4067/S0718-50062010000100003, Formación Universitaria, 3(1), $11-20$ (2010)

Nadelson L.S., Heddy B.C. y otros tres autores, Conceptual Change in Science Teaching and Learning: Introducing the Dynamic Model of Conceptual Change, doi:10.17583/ijep.2018.3349, Int. J. Educational Psychology 7(2), 151-195 (2018)

Prosser, M., Trigwell, K. y otros dos autores, Students' experiences of Studying Physics Concepts: The Effects of Disintegrated Perceptions and Approaches, doi.org/10.1007/BF03173167, European Journal of Psychology of Education, $15(1), 61-74(2000)$

SanMarti, N., El Diseño de Unidades Didácticas. En Palacios, F. J. y León, P.C., Didáctica de las ciencias experimentales, ISBN 84-268-1051-9, Alcoy, Editorial Marfil, España (2002)

Shaw, G.S., Interdisciplinary Teaching Training in Higher Education: An Evaluation Proposal, dx.doi.org/10.22347/21752753v12i34.2279, Meta: Avaliacao, 12(34), 181-210 (2020)

Taber K. S. Alternative Conceptions and the Learning of Chemistry, doi.org/10.1002/ijch.201800046, Israel Journal of Chemistry, 59 (6), 450-469 (2019)

Toro-Carvajal, L.A., Ortiz-Álvarez, H.H. y otros dos autores, doi:10.5294/edu.2012.15.2.1, Los Sistemas Cognitivos Artificiales en la Enseñanza de la Matemática, Educación y Educadores, 15(2), 167-183 (2012)

Yang, Y.F. y Kuo, N.C., New Teaching Strategies from Student Teachers' pedagogical Conceptual Change in CALL, doi.org/10.1016/j.system.2020.102218, System, 90,102218, 1-12 (2020) 
